\title{
Schlaganfallprophylaxe bei Vorhofflimmern Wann Sie Ihre Patienten von Marcumar überzeugen müssen
}

— "Muss ich denn unbedingt Marcumar nehmen, Herr Doktor? Reicht Aspirin nicht aus?" fragen viele Patienten mit Vorhofflimmern, wenn sie erfahren, dass aufgrund ihrer Risikokonstellation ASS zur Blutgerinnungshemmung nicht mehr genügt. Die richtige ärztliche Antwort: „Mit dem Vitamin-K-Antagonisten wird im Schnitt die Zahl der Schlaganfälle um $62 \%$ verringert. Mit ASS allein wird eine Verringerung von minus $22 \%$ erreicht." Dies ist jedenfalls die Antwort von Prof. Markus Haass aus Mannheim, die er beim Mannheimer Pressekreis von Roche Diagnostics empfohlen hat. Er wies auch darauf hin, dass ein Vorhofflimmern bei Patienten über 75 Jahren das Risiko für einen Schlaganfall um das Drei- bis Siebenfache erhöht.

Zur Beurteilung der Risikokonstellation wird der CHADS2-Score genutzt, erläuterte Haas. Risikofaktoren sind Herzinsuffizienz ( $C=$ Congestive Heart failure), Hypertonie (H), Alter über 75 Jahre (A), Diabetes mellitus (D), Schlaganfall oder
TIA in der Anamnese (S). Sind zwei dieser Faktoren gleichzeitig vorhanden gehört der Vitamin-K-Antagonist zur Prophylaxe dazu. Die „2“ im Akronym des Scores steht für die doppelte Gewichtung des Schlaganfalls oder einer TIA in der Anamnese. Liegt bei Vorhofflimmern dieser Risikofaktor vor, soll auf jeden Fall mit Marcumar antikoaguliert werden. In einer Neu-

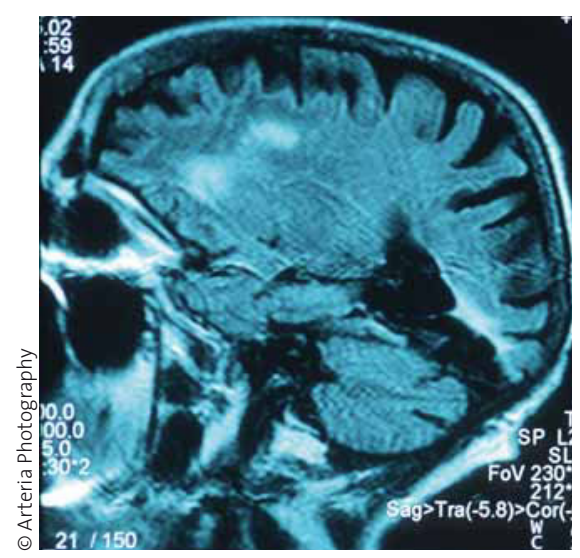

Jeder fünfte Schlaganfall ist durch Vorhofflimmern verursacht. fassung der Leitlinie durch das ESC, die für Ende 2010 erwartet wird, werden auch die Faktoren Mitralstenose, KHK, künstlicher Klappenersatz oder Thyreotoxikose Berücksichtigung finden.

\section{Individuelles Gerinnungsmanagement}

Haass erinnerte auch daran, dass es zu Beginn einer Marcumarisierung zur paradoxen Übergerinnung kommen kann, weshalb beim Start in aller Regel gleichzeitig mit Heparinen behandelt wird. Der prognostische Nutzen der Antikoagulation sei mit der Einhaltung des optimalen Gerinnungsbereiches (Ziel-INR 2,0-3,0) verknüpft. Bei zu geringer Gerinnungshemmung $(I N R<2,0)$ besteht kein adäquater Schutz. Bei zu starker Gerinnungshemmung (INR > 3,0) ist das Blutungsrisiko erhöht. Auch sei individuell angepasstes Vorgehen starren INR-Kontrollen alle drei bis vier Wochen überlegen.

\footnotetext{
- Rainer Klawki

Quelle: Mannheimer Pressekreis, 18. Juni 2010 (Veranstalter: Roche diagnostics)
}

\section{Analgetikum mit neuem Wirkmechanismus in Sicht}

- Noch für dieses Jahr wird die Zulassung eines neuartigen Analgetikums erwartet: Tapentadol ist der erste Vertreter der Substanzklasse der MOR-NRI. Die Substanz kombiniert $\mu$-Opioid-Rezeptor-(MOR-)Agonismus und Noradrenalin-Wiederaufnahme-Hemmung (NRI) in einem Molekül. In synergistischer Weise wird die Schmerzwahrnehmung unterdrückt und körpereigene schmerzhemmende Mechanismen werden aktiviert. Gleichzeitig werden über den $\mu$-Rezeptor vermittelte $\mathrm{Ne}$ benwirkungen reduziert.

\section{Sinnvolle Option bei Mixed Pain} In Phase-III-Studien mit Tapentadol wurden bislang über 7000 Patienten mit starken Schmerzen eingeschlossen, bei mehr als der Hälfte waren die Schmerzen chronisch. Aus dem umfangreichen Studienprogramm geht hervor, dass sich die Substanz in einem breiten Indikationsspektrum einsetzen lässt. Da Tapentadol sowohl bei nozizeptiven als auch bei neuropathischen Schmerzen wirkt, erscheint es besonders geeignet für die Behandlung gemischter Schmerzsyndrome wie z. B. Kreuzschmerzen. In einer doppelblinden placebo- und aktivkontrollierten Phase-III-Studie bei Patienten mit lumbalen Rückenschmerzen zeigte Tapentadol eine vergleichbare analgetische Wirksamkeit wie Oxycodon - bei deutlich geringeren Nebenwirkungen. Die Abbruchraten aufgrund von gastrointestinalen Ereignissen betrugen unter Tapentadol 5,3\% und unter Oxycodon
18,3\% (Buynak R et al., APS Congress, San Diego, 2009).

Tapentadol wurde als retardierte Form (PR) zur Behandlung starker chronischer Schmerzen entwickelt. Im Juni 2009 hat Grünenthal die Marktzulassung für Tapentadol bei mehreren europäischen Zulassungsbehörden, einschließlich BfArM, beantragt. Die Markteinführung für Deutschland wird für das vierte Quartal 2010 erwartet. Tapentadol in Retardform wird voraussichtlich in den Wirkstärken 50, 100, 150, 200 und 250 mg zur Verfügung stehen. Das Dosisintervall ist zweimal täglich.

\section{- Rainer Bubenzer}

Quelle: Bericht vom Zukunftsforum Schmerz, Berlin, 4. Juni 2010 (Veranstalter Grünenthal $\mathrm{GmbH}$ ) 